\title{
Equivocal usefulness of FDG for the noninvasive imaging of abdominal aortic aneurysms
}

\author{
Laurent M. Riou • Gérald Vanzetto • Alexis Broisat • \\ Daniel Fagret • Catherine Ghezzi
}

Published online: 25 September 2014

(C) Springer-Verlag Berlin Heidelberg 2014

\begin{abstract}
Abdominal aortic aneurysms (AAA) usually progress asymptomatically and are undetected until rupture occurs. Once ruptured, the mortality rate of AAA is about $80 \%$. Among all patients with ruptured AAA, about one-third die without reaching hospital, and about another quarter reach hospital but die prior to surgical intervention. Among the remaining patients (about $40 \%$ ) surviving AAA rupture long enough to benefit from surgical intervention, the perioperative mortality has historically reached about $50 \%$ [1], a value that has however recently decreased owing to the introduction of endovascular repair [2]. On the other hand, elective AAA repair has a much lower in-hospital mortality rate, with recently reported values of $1.3 \%$ and $4.7 \%$ for endovascular and open surgical repair, respectively [3], thereby strongly emphasizing the need for screening and identification of those patients with prone-to-rupture AAA.

The crude estimate of AAA diameter remains the most widely used predictive risk factor for AAA rupture and the sole therapeutic management criterion. Indeed, in both the 2014 European Society of Cardiology and the 2011 American College of Cardiology/American Heart Association guidelines, aortic repair is a class I indication if the AAA diameter exceeds $55 \mathrm{~mm}[4,5]$. However, the limitations of this parameter are well acknowledged, and it is therefore unanimously agreed that a more robust noninvasive predictor of the individual risk of AAA rupture is required. Although being much
\end{abstract}

\footnotetext{
L. M. Riou $(\bowtie) \cdot$ G. Vanzetto · A. Broisat • D. Fagret • C. Ghezzi INSERM, U1039, Radiopharmaceutiques Biocliniques Grenoble, France, Université de Grenoble, UMR-S1039, Grenoble 38000, France

e-mail: laurent.riou@ujf-grenoble.fr

G. Vanzetto

Cardiology University Clinic, Grenoble 38000, France

D. Fagret

Nuclear Medicine University Clinic, Grenoble 38000, France
}

less documented than atherogenesis, the current knowledge regarding the pathophysiology of AAA indicates that parietal inflammation, matrix degradation and smooth muscle cell apoptosis are required for AAA pathogenesis [6]. FDG has been suggested as a potentially useful agent for the molecular imaging of vascular inflammation due to the elevated carbohydrate metabolism of extravasated inflammatory cells [7]. As such, the tracer has been extensively evaluated in both the experimental and clinical settings for the noninvasive imaging of inflammatory processes in vulnerable atherosclerotic lesions as well as in AAA.

In the multicentre study by Barwick and colleagues published in this issue of the European Journal of Nuclear Medicine \& Molecular Imaging [8], the authors used PET imaging of FDG uptake to retrospectively compare the metabolic activity in the abdominal aortic wall of 151 patients with AAA (mean aortic diameter, $50 \pm 13 \mathrm{~mm}$ ) with that of 159 individuals with no AAA (mean aortic diameter, $21 \pm 3 \mathrm{~mm}$ ) and matched for age, sex, diabetes, smoking status, statin use and indication for PET/CT. This cohort is the largest so far used for the comparison of FDG uptake between AAA and normal vessels in well-matched patients. FDG uptake was analysed by semiquantitative visual scoring as well as quantitatively using SUVmax and target to background ratios utilizing mediastinal blood pool or descending thoracic aorta activities for normalization. Although the retrospective nature and the design of the study limit the conclusions that can be drawn with respect to the potential of FDG for predicting AAA growth or rupture, the results unequivocally show that there was no correlation between AAA size and FDG uptake and that there was no statistically significant difference in FDG uptake between AAA and normal abdominal aortas.

The study by Barwick et al. therefore joins the ranks of those suggesting the suboptimal usefulness of FDG for AAA evaluation, which face the ranks of previously published studies that reached opposite conclusions by suggesting the 
promising potential of the tracer in this indication (see Buijs et al. [9] as well as Table 1 from Barwick et al. [8]). In addition to the lack of a standardized methodology for the analysis of FDG PET images of AAA tracer activity, the reasons for such equivocal conclusions may originate from several sources related to the mechanisms of both AAA pathophysiology and FDG uptake:

1. FDG being a tracer of general carbohydrate metabolic activity, it may lack specificity and be taken up by all metabolically active cells such as endothelial cells, smooth muscle cells, neutrophils and lymphocytes, in addition to macrophages, parietal infiltration of which is considered a reliable marker of vascular wall inflammation $[10,11]$.

2. As observed in atherosclerotic lesions in which M1/M2 macrophage polarization occurs resulting in the presence of a continuum of proatherogenic and antiatherogenic cells in atherosclerotic plaques [12], an imbalance in M1/M2 macrophages has been observed in ruptured vs. unruptured human cerebral aneurysms [13]. Should these findings be replicated in AAA, FDG imaging would not be likely to allow the differentiation between proinflammatory (M1) and antiinflammatory (M2) macrophages, thereby reducing its relevance for the identification of prone-torupture aneurysms.

3. Perivascular adipose tissue [14] might also represent a potential confounding factor for FDG aortic imaging, regardless of whether tracer uptake is mediated by adipose tissue metabolism itself $[15,16]$ or by inflammatory cell infiltrates resulting from the proinflammatory phenotype of perivascular adipocytes [17].

4. Importantly, the results of a clinical retrospective longitudinal study have indicated that areas of focal arterial FDG uptake are transient [18]. In parallel, the natural history of AAA development probably involves phases of inflammation and expansion. Ultimate AAA rupture due to collagen breakdown and rupture has been shown to be closely related to increased MMP expression and medial neovascularization with both phenomena being unrelated to the inflammatory infiltrate $[19,20]$, suggesting that AAA wall inflammation as assessed by FDG uptake might not necessarily indicate the imminence of AAA rupture.

5. Finally, aortic aneurysm expansion - and eventually rupture - might result from both predisposing factors (e.g. atherosclerosis, inflammation, matrix remodelling) and precipitating factors (haemodynamics, abrupt blood pressure increase, exacerbation of inflammation secondary to infection, etc.) as strongly suggested for vulnerable coronary plaque rupture [21]. The approach involving solely predisposing factors - even using the most accurate technique - may therefore fail to predict the clinical outcome of the disease.
Taken together, these results suggest that FDG as a tracer of metabolically active inflammatory cells might not be optimally suited to the molecular imaging of prone-to-rupture AAA. Considering the equivocal clinical usefulness of the mere assessment of vascular wall inflammation for the diagnostic and prognostic evaluation of AAA that was previously suggested and that is further shown by the present study of Barwick et al. [8], the development of methodologies such as SPECT/CT, PET/CT and MRI allowing simultaneous anatomical and molecular imaging of the AAA wall with agents targeting potentially more specific biomarkers of prone-torupture AAA than FDG [22-24] might therefore be a relevant area of research for the ultimate noninvasive prediction of AAA growth and rupture.

\section{References}

1. Reimerink JJ, van der Laan MJ, Koelemay MJ, Balm R, Legemate DA. Systematic review and meta-analysis of population-based mortality from ruptured abdominal aortic aneurysm. Br J Surg. 2013;100: $1405-13$.

2. Qin C, Chen L, Xiao YB. Emergent endovascular vs. open surgery repair for ruptured abdominal aortic aneurysms: a meta-analysis. PLoS One. 2014;9(1):e87465.

3. Stather PW, Sidloff D, Dattani N, Choke E, Bown MJ, Sayers RD. Systematic review and meta-analysis of the early and late outcomes of open and endovascular repair of abdominal aortic aneurysm. Br J Surg. 2013;100:863-72.

4. Erbel R, Aboyans V, Boileau C, Bossone E, Di Bartolomeo R, Eggebrecht H, et al. 2014 ESC Guidelines on the diagnosis and treatment of aortic diseases: Document covering acute and chronic aortic diseases of the thoracic and abdominal aorta of the adult. The Task Force for the Diagnosis and Treatment of Aortic Diseases of the European Society of Cardiology (ESC). Eur Heart J. 2014. doi:10. 1093/eurheartj/ehu281

5. Rooke TW, Hirsch AT, Misra S, Sidawy AN, Beckman JA, Findeiss L, et al. American College of Cardiology Foundation Task Force; American Heart Association Task Force. Management of patients with peripheral artery disease (compilation of 2005 and 2011 ACCF/AHA Guideline Recommendations): a report of the American College of Cardiology Foundation/American Heart Association Task Force on Practice Guidelines. J Am Coll Cardiol. 2013;61:1555-70.

6. Nordon IM, Hinchliffe RJ, Loftus IM, Thompson MM. Pathophysiology and epidemiology of abdominal aortic aneurysms. Nat Rev Cardiol. 2011;8:92-102.

7. Tarkin JM, Joshi FR, Rudd JH. PET imaging of inflammation in atherosclerosis. Nat Rev Cardiol. 2014;11:443-57.

8. Barwick TD, Lyons OT, Mikhaeel NG, Waltham M, O'Doherty MJ. 18F-FDG PET-CT uptake is a feature of both normal diameter and aneurysmal aortic wall and is not related to aneurysm size. Eur J Nucl Med Mol Imaging. 2014. doi:10.1007/s00259-014-2865-9.

9. Buijs RV, Willems TP, Tio RA, Boersma HH, Tielliu IF, Slart RH, et al. Current state of experimental imaging modalities for risk assessment of abdominal aortic aneurysm. J Vasc Surg. 2013;57: 851-9.

10. Rosenbaum D, Millon A, Fayad ZA. Molecular imaging in atherosclerosis: FDG PET. Curr Atheroscler Rep. 2012;14:429-37.

11. Naghavi M, Libby P, Falk E, Casscells SW, Litovsky S, Rumberger J, et al. From vulnerable plaque to vulnerable patient: a call for new 
definitions and risk assessment strategies: Part I. Circulation. 2003;108:1664-72.

12. Leitinger N, Schulman IG. Phenotypic polarization of macrophages in atherosclerosis. Arterioscler Thromb Vasc Biol. 2013;33:1120-6.

13. Hasan D, Chalouhi N, Jabbour P, Hashimoto T. Macrophage imbalance (M1 vs. M2) and upregulation of mast cells in wall of ruptured human cerebral aneurysms: preliminary results. J Neuroinflammation. 2012;9: 222. 5 .

14. Thanassoulis G, Massaro JM, Corsini E, Rogers I, Schlett CL, Meigs $\mathrm{JB}$, et al. Periaortic adipose tissue and aortic dimensions in the Framingham Heart Study. J Am Heart Assoc. 2012;1:e00885.

15. Toczek J, Broisat A, Perret P, Desruet MD, Fagret D, Riou LM, et al. Periaortic brown adipose tissue as a major determinant of $\left[{ }^{18} \mathrm{~F}\right]-$ fluorodeoxyglucose vascular uptake in atherosclerosis-prone, apoE-/- mice. PLoS One. 2014;9:e99441.

16. Sacks H, Symonds ME. Anatomical locations of human brown adipose tissue: functional relevance and implications in obesity and type 2 diabetes. Diabetes. 2013;62:1783-90.

17. Omar A, Chatterjee TK, Tang Y, Hui DY, Weintraub NL. Proinflammatory phenotype of perivascular adipocytes. Arterioscler Thromb Vasc Biol. 2014;34:1631-6.

18. Menezes LJ, Kayani I, Ben-Haim S, Hutton B, Ell PJ, Groves AM. What is the natural history of $18 \mathrm{~F}-\mathrm{FDG}$ uptake in arterial atheroma on $\mathrm{PET} / \mathrm{CT}$ ? Implications for imaging the vulnerable plaque. Atherosclerosis. 2010;211:136-40.
19. Wilson WR, Anderton M, Schwalbe EC, Jones JL, Furness PN, Bell PR, et al. Matrix metalloproteinase- 8 and -9 are increased at the site of abdominal aortic aneurysm rupture. Circulation. 2006;113:43845.

20. Choke E, Thompson MM, Dawson J, Wilson WR, Sayed S, Loftus $\mathrm{IM}$, et al. Abdominal aortic aneurysm rupture is associated with increased medial neovascularization and overexpression of proangiogenic cytokines. Arterioscler Thromb Vasc Biol. 2006;26: 2077-82.

21. Arbab-Zadeh A, Nakano M, Virmani R, Fuster V. Acute coronary events. Circulation. 2012;125:1147-56.

22. Botnar RM, Wiethoff AJ, Ebersberger U, Lacerda S, Blume U, Warley A, et al. In vivo assessment of aortic aneurysm wall integrity using elastin-specific molecular magnetic resonance imaging. Circ Cardiovasc Imaging. 2014;7:67989.

23. Bonnard T, Yang G, Petiet A, Ollivier V, Haddad O, Arnaud D, et al. Abdominal aortic aneurysms targeted by functionalized polysaccharide microparticles: a new tool for SPECT imaging. Theranostics. 2014;4:592-603.

24. Klink A, Heynens J, Herranz B, Lobatto ME, Arias T, Sanders HM, et al. In vivo characterization of a new abdominal aortic aneurysm mouse model with conventional and molecular magnetic resonance imaging. J Am Coll Cardiol. 2011;58:2522-30. 